Instituto Internacional de Investigación y Desarrollo Tecnológico Educativo INDTEC, C.A.

DOI: $h$ ttps://doi.org/10.29394/Scientific.issn.2542-2987.2020.5.15.17.336-349

OAI-PMH: http://www.indteca.com/ojs/index.php/Revista Scientific/oai

Ensayo Original / Original Essay

\title{
Evaluación de Desempeño como Factor Estratégico para Rendimiento Académico del Docente Universitario
}

Autor: Luís Alberto Ortiz López Universidad Latinoamericana y del Caribe, ULAC luiyialberto64@hotmail.com Yaracuy, Venezuela https://orcid.org/0000-0002-2803-008X

\section{Resumen}

El sector universitario debe estar a la vanguardia de los desafíos y cambios que se producen en una sociedad globalizada, tener perspectiva hacia dónde dirigir sus acciones y planear hacia el futuro, el cual le permita razonar en detalle su misión, visión, metas, estrategias y actividades que se requieren para cumplir con los objetivos deseados, al mismo tiempo se debe enfrentar los diferentes retos que se producen cotidianamente en los ambientes de enseñanza y aprendizaje. De modo tal, que este ensayo, pretende hacer una reflexión con respecto al beneficio que produce la evaluación de desempeño en el trabajador y las ventajas que tiene para los supervisores universitarios sobre el aprovechamiento de las posibilidades materiales y humanas que conlleva a facilitar y agrupar aún más a todos los funcionarios en torno a las metas establecidas en el sector universitario. De allí que, que la evaluación de desempeño mediante el factor estratégico viene a representar un mecanismo efectivo a la organización universitaria porque permite precisar los roles y responsabilidades de cada uno de los docentes y así poder obtener un mayor impacto y efectos positivos en el rendimiento académico de sus funciones en beneficio de la sociedad.

Palabras clave: evaluación del docente; estrategias educativas; rendimiento académico.

Cómo citar este ensayo:

Ortiz, L. (2020). Evaluación de Desempeño como Factor Estratégico para Rendimiento Académico del Docente Universitario. Revista Scientific, 5(15), 336-349, e-ISSN: 2542-2987. Recuperado de: https://doi.org/10.29394/Scientific.issn.2542-2987.2020.5.15.17.336-349

Fecha de Recepción:

29-07-2019
Fecha de Aceptación:

03-10-2019
Fecha de Publicación: 05-02-2020 


\title{
Performance Evaluation as a Strategic Factor for Academic Performance of University Teachers
}

\begin{abstract}
The university sector must be at the forefront of the challenges and changes that occur in a globalized society, have perspective on where to direct its actions and plan for the future, which allows it to reason in detail its mission, vision, goals, strategies and activities that are required to meet the desired objectives, at the same time the different challenges that occur daily in the teaching and learning environments must be faced. Thus, this essay intends to reflect on the benefit produced by the evaluation of worker performance and the advantages it has for university supervisors over the use of material and human possibilities that leads to facilitate and group even more to all officials around the goals set in the university sector. Hence, that the evaluation of performance through the strategic factor comes to represent an effective mechanism to the university organization because it allows to specify the roles and responsibilities of each of the teachers and thus be able to obtain a greater impact and positive effects on academic performance of its functions for the benefit of society.
\end{abstract}

Keywords: teacher evaluation; educational strategies; academic performance.

Date Received:

29-07-2019
Date Acceptance:

03-10-2019
Date Publication: 05-02-2020 


\section{Introducción}

En los últimos años, se ha acrecentado la investigación sobre la gestión del conocimiento y cada vez más tiene relevancia dentro de las organizaciones, de allí que es importante tomar en cuenta los factores estratégicos que permiten evaluar este conocimiento en el ser humano. De manera que, el factor estratégico dentro de la gestión de recursos humanos es considerado como el mecanismo para la evaluación de desempeño porque puede ser utilizada como instrumento en la verificación del grado de desempeño de los objetivos formulados a nivel individual que favorece al desarrollo laboral.

Dicho de otra manera, cuando se realiza el proceso de evaluación de desempeño, el procedimiento admite a la organización obtener información para las tomas de decisiones, ya que a través de este proceso le permite al evaluador evidenciar cuando los resultados son desfavorable realizar mecanismo correctivos que le acceda a la organización y llegar alcanzar los objetivos planteados, por lo contrario, cuando estos resultados son favorable para la institución y para el trabajador estos debe ser alentado por el evaluador.

En este sentido, la evaluación de desempeño, constituye para trabajador, un instrumento de calificación que permite co-evaluarse con respecto a sus tareas asignadas por el evaluador, asimismo a través de ella, se puede evidenciar las fortaleza y debilidades que posee el funcionario al momento de ejecutar sus funciones.

Ahora bien, desde el contexto universitario la evaluación de desempeño, representa el mecanismo que constituye para las partes involucradas, un instrumento de primer orden, ya que el docente universitario al saberse evaluado, responde inmediatamente a un mejor desempeño, mayor rendimiento que redunda en el rendimiento académico y en la eficacia de la institución.

De allí que, este ensayo se centra en el proceso de evaluación del 
desempeño, siendo aprovechado como soporte para las autoridades correspondientes y para que logren el desarrollo del personal universitario a través de la aplicación de este proceso, con el fin de solucionar los problemas que están presentes y al mismo tiempo preverlos para un futuro.

\section{Desarrollo}

El recurso humano es considerado una fuerza de gran importancia, y será más que nunca el elemento clave para el logro de objetivos de cualquier organización, con esto quiero decir, que personal que labora en una organización específicamente en el ámbito universitario es el principal instrumento para alcanzar los objetivos planteados, por lo tanto para llegar a lograrlo el trabajador universitario debe pasar por un proceso de evaluación de desempeño que permita identificar aspectos importantes en el desenvolvimiento, entre los cuales se pueden mencionar la autocorrección, apariencia, actitud, habilidad y destreza para la planeación y creatividad, que son elementos capaz de neutralizar la subjetividad para un mejor desempeño académico.

Debido a esto, las organizaciones educativas universitarias de hoy en día son más vanguardistas en cuanto a la búsqueda de técnicas innovadoras que permitan lograr la integración del recurso humano para el cumplimiento de las metas trazadas y que perduren en el tiempo. Dentro de este contexto, la evaluación de desempeño representa en las organizaciones universitarias un proceso que depende de la función del evaluador y del docente universitario, ya que ambos constituyen un recurso valioso, importante y generador de conocimiento estratégicos que generan resultados propios de una labor. De allí que, Carpio (2014), señala que: "la evaluación del desempeño constituye una técnica de apreciación de un individuo en el cargo o puesto y de su potencial de desarrollo en la organización" (pág. 63); por eso, este proceso se utiliza para estimar y juzgar, el valor, la excelencia, las aptitudes y cualidades 
del docente universitario con respecto a sus funciones a nivel académico.

Por otra parte, se puede decir que la evaluación del desempeño es un concepto dinámico que constituye una herramienta de dirección imprescindible en la actividad administrativa. De ahí, que Chiavenato (2014), señala que: "los resultados de la evaluación del desempeño son las evidencias plasmadas en el uso de las técnicas que determina las necesidades de los trabajadores, de la organización, así como el cumplimiento de sus funciones" (pág. 88). De esta manera los docentes universitarios han de tener conocimientos acerca de las tareas y funciones a cumplir, con el fin de que su evaluación de su desempeño sea positiva.

Conviene subrayar en este punto, que en la actualidad, el evaluador universitario hace esfuerzos por determinar hasta qué punto sus docentes están cumpliendo con las actividades académicas y los objetivos planteado, sin embargo, esto no es suficiente ya que deben utilizarse por parte del evaluador diferentes variaciones de estos procedimientos para mejorar la objetividad y asegurar la precisión de un proceso de evaluación del desempeño más eficaz. Además, es bueno indicar que la evaluación de desempeño no solo beneficiara al docente universitario con incentivos, sino que les brindara la oportunidad de reconocer sus propias necesidades y expectativas personales, así como el rendimiento académico en el momento de impartir los procesos de enseñanza y aprendizaje a sus estudiantes.

De manera que, para la realización de una evaluación de desempeño eficaz, el evaluador universitario debe tomar en cuenta que los docentes tienen expectativas diferentes acerca del trabajo académico. Sin embargo, la evaluación de desempeño debe tomarse como factor estratégico en la educación universitaria de forma sistemática y formal que permite detectar problemas en el personal a nivel del análisis y evaluaciones con una visión propia de la integración dentro de ellos. 


\subsection{Educación Universitaria}

El desenvolvimiento del desempeño laboral de un profesor universitario exige satisfacer las expectativas de la institución educativa, Por esto, Peña (2017), establece que: "los docentes desde sus diversos ambientes hacen necesaria una constante formación que les permita reforzar la orientación e inducción tanto en los modelos de innovación curricular, como a la calidad de la profesión y al aprendizaje" (pág. 137). De manera que, para que el docente universitario este en constante formación es necesario que sea evaluado de acuerdo a sus funciones, obligaciones y responsabilidades asignadas por parte de los evaluadores en cargos específicos, que permita al profesional universitario contribuir al fortalecimiento del trabajo que realiza.

Es por ello, que en nuestro contexto universitario se hace necesario el adiestramiento y capacitación del profesional de la docencia, donde este presto a enfrentar los desafíos y cambios que exige actualmente la sociedad tan globalizada. Por consiguiente, las universidades deben estar prestas a enfrentar los diferentes cambios significativos que se produce dentro y fuera de estos recintos universitarios, con la finalidad de ser tomado en cuenta al momento de ser evaluado el trabajador por el supervisor inmediato.

En este sentido, los docentes universitarios deben poseer conocimiento sobre la evaluación de desempeño, que le permita romper con el paradigma que este proceso solo sirve para el docente facilitar una cátedra y el estudiante en obtener un título, sino que vaya más allá de esto, que le conlleve alcanzar las metas propias y colectivas para todos los involucrados.

Bajo esta perspectiva, es responsabilidad colectiva para todo aquellos que hacen vida activa dentro del sector universitario en mejorar el nivel académico del estudiantado, se debe reflexionar con respecto a nuestras funciones como docente $y$ ofrecer diferentes alternativa que permita al estudiante tener un abanico de oportunidades al momento del proceso de enseñanza y aprendizaje, con la oportunidad de repotenciar la capacidad de 
razonar, argumentar y desarrollar habilidades que favorezca la convivencia entre el docente y el estudiante.

Es por ello, que la educación universitaria esta llamada a estar a la par de los nuevos mecanismo y procesos de evaluación de desempeño de su personal a su cargo, donde le permita al docente generar teorías y conceptos, cambiando los paradigmas tradicionales dentro y fuera del ambiente de clase ya que en la actualidad esto obstaculizan el mejor desempeño en el estudiante con respecto a su rendimiento académico.

Dicho de otra manera, y desde mi acción profesional el proceso de evaluación de desempeño representa para el sector universitario un mecanismo que permite obtener y conocer como somos en nuestro ámbito laboral y donde podemos llegar, dándonos la libertad para tomar nuestras propias decisiones con mayor fundamento en beneficio del colectivo.

Como puede observarse, la educación universitaria debe ofrecer a sus trabajadores especialmente al docente mayor alternativa al momento de ser evaluado, así como desencadenar las acciones pertinentes, permitiéndole dar un conocimiento interior que vas más allá de las perspectivas de la formación integral, donde el docente cumpla su rol de orientador en el sentido de convertir en fortaleza las debilidades, así como también indagar, inferir y comprender de manera positiva y que internalice que es parte de un proceso de evaluación de desempeño, donde se busca es la construcción de manera efectiva e innovadora a la solución de problema que afecta al proceso de enseñanza y aprendizaje de la sociedad estudiantil.

\subsection{Evaluación de Desempeño}

De acuerdo a la administración de recursos, la evaluación del desempeño es un tema muy explícito y necesario en todas las organizaciones especialmente la universitaria. En este sentido, el proceso de evaluación desde el contexto universitario para ser efectivo debe contar con todos el 
personal que hacen vida activa dentro de la organización. De allí que, tanto los evaluadores como los evaluados debe estar presto a contribuir al logro de los objetivos institucionales.

Cabe destacar que la mayor parte de las organizaciones universitarias aplican una evaluación de desempeño para determinar el nivel de responsabilidad del docente desde el punto de vista académico y para un ascenso de puesto de trabajo, es por ello que luego de haber aplicado el proceso de evaluación, se debe tomar la decisión de comunicarle al evaluado sobre los resultados encontrado cuyo objetivo es alcanzar las metas propuesta por la organización universitaria. Sin embargo, para estimular la decisión en cuanto al buen desempeño, el docente universitario debe estar en constante retroalimentación de manera continua, evidenciando de manera objetiva el trabajo realizado y lo que necesita para mayor desenvolvimiento y así medir el cumplimiento de sus funciones.

Al respecto, Milkovich y Boudreaw (2016), se refieren al desempeño del empleado como: "el grado en el cual los trabajadores cumplen los requisitos del cargo" (pág. 96). De manera que, la ocupación se manifiesta en las acciones observadas, relevantes para los objetivos de la organización y que puedan ser medidas en términos de las competencias de cada docente universitario y su nivel de contribución a la institución.

Es por ello, que en todo proceso de evaluación es determinante establecer esquemas con previsión de los instrumentos a aplicar, ya que son ellos los que determinarán el fracaso o el éxito del proceso. Todo lo que se planifique al respecto, debe ser comunicado al personal evaluado de manera que comprendan su propósito y su importancia tanto para ellos como para la organización donde prestan sus servicios. Por lo cual solamente a través de una extraordinaria planificación y por medio de un razonamiento cuidadoso de la evaluación del desempeño, puede ser estimada el rango y la situación de las calificaciones, lo cual se logra a través de un mecanismo adecuado donde 
se utilice los datos disponibles, que existan en la organización universitaria.

Como ya se ha aclarado, para que institución universitaria utilice efectivamente la competencia y talento que posee su personal docente debe evaluarlo en forma continua y vigilar así su progreso, utilizando mecanismos de observación, con el objetivo de evidenciar el desenvolvimiento del evaluado, al momento de ejecutar sus tareas diarias y poder hacer los correctivos necesarios en beneficio del buen funcionamiento de las actividades asignadas por la organización universitaria.

\subsection{Factores Estratégicos de Evaluación de Desempeño}

El factor estratégico según David (2014): es "la formulación, ejecución y evaluación de acciones que permitirán que una organización logre sus objetivos" (pág. 3). De este modo, el factor estratégico es uno de los métodos más populares porque no sólo se centran en las fortalezas y debilidades internas, sino también en el entorno externo en el que la institución universitaria está operando.

Por otra parte, Serna (2008), la define como: "el proceso mediante el cual quienes toman decisiones en una organización obtienen, procesan y analizan información pertinente interna y externa, para evaluar la situación presente de la empresa y decidir sobre el direccionamiento hacia el futuro" (pág. 55).

Esto significa, que el factor estratégico es una herramienta por excelencia de la gerencia estratégica en el contexto universitario, cuyo objetivo consiste en la búsqueda de una o más ventajas competitivas de la institución, permitiendo crear o preservar sus ventajas, todo esto en función de la misión y los objetivos establecidos por el sector universitario.

Por consiguiente, los factores estratégicos de evaluación permiten conocer el desempeño del trabajador, es decir su actuación. Además, en toda evaluación del desempeño se toman en cuenta diferentes aspectos por medio 
de los cuales se evaluará al personal a través de diferentes indicadores que son reseñados como factores de evaluación.

Entre los factores de gran relevancia, se considera la Capacidad, que viene a contribuir al desarrollo de las actividades por los docentes universitarios, lo cual, los lleva a elevar el rendimiento académico entre los estudiantes, el Conocimiento, es otro factor estratégico en toda evaluación de desempeño independientemente del cargo que ocupe dentro de las organización universitaria, el docente debe poseer los conocimientos previos de las tareas a ejecutar en los recitos universitarios, específicamente debe saber que actividades académicas se realizan como lo hace y cuáles son las estrategias pedagógicas que utilizan para lograr los objetivos planeados.

Asimismo, otro de los factores estratégicos es la Puntualidad, que contribuye en el docente universitario realizar sus actividades en el momento preciso. Cabe destacar, que la puntualidad es la disciplina de un trabajador de llegar a tiempo y de organizar sus tareas diarias a ejecutar. $Y$ es, sin dudas, una norma básica de convivencia en sociedades, donde el tiempo vale.

Otro de los factores estratégico es la Creatividad, donde se pone de manifiesto nuevas ideas o conceptos, que el docente experimenta en el ambiente de aprendizaje con sus estudiantes para lograr los objetivos de innovación y cambio que se proponga la organización. Universitaria.

Por último y no menos importante, tenemos la Cooperación, donde el evaluador y el evaluado en conjunto con todos los que hacen vida activa en los recintos universitarios, colaboren entre sí, para lograr fines comunes. Así pues, cada docente universitario debe poner lo mejor de sí mismo para el bien de todos especialmente del estudiantado. Conviene subrayar en este punto que este factor estratégico nadie quiere ganar individualmente sino beneficiarse en conjunto fomentando la cooperación y contribuir a mejorar su desempeño y el de la organización. 


\subsection{Rendimiento Académico}

El Rendimiento Académico es considerado como el fruto del aprovechamiento del contenido de los planes de estudio, formulado en calificaciones dentro de una escala convencional. En otras palabras, se refiere al resultado numéricos atreves de exposiciones, mesas de trabajos informe escrito, ensayos, socializaciones entre otros que se obtiene en el proceso de aprendizaje de conocimientos, relacionado a las evaluaciones que realiza el docente mediante encuentros programados $y$ otras actividades complementarias.

Dicho de otra manera, el Rendimiento académico representa para el sector universitario una punta de lanza que puede ser cristalizada mediante los procesos de evaluación de desempeño como factor estratégico. Conviene subrayar que este mecanismo de evaluación no se trata de que el docente universitario obligue al estudiante a memorizar el aprendizaje sino de cuanto han incorporado realmente a su conocimiento, evidenciando mediante el rendimiento académico su manera de sentir, de resolver los problemas y hacer o utilizar cosas aprendidas.

\section{Conclusión}

Al concluir el presente ensayo, se puede visualizar a través de su desarrollo, que para evaluar el desempeño del trabajador especialmente desde el ámbito universitario, deben tomarse en consideración aspectos involucrados en el contexto laboral donde se desenvuelve, así como también la variedad de características físicas, mentales y emocionales que posee cada docente. Dicho de otra manera, se debe tomar en cuenta en cada docente universitario su desarrollo, su preparación y capacitación académica, así como sus expectativas, sus necesidades y su forma de impartir su clase en el ambiente de aprendizaje, para que exista una objetividad al momento de ser evaluarlo. 
Por otra parte, es necesario que el evaluador universitario tenga presente al momento del procedimiento las funciones, obligaciones y responsabilidades asignadas al evaluado dentro de la organización universitaria, lo cual contribuirá a mejorar el trabajo realizado. Cabe agregar, para aplicar la evaluación de desempeño como factor estratégico, se debe atender a las debilidades reflejadas en cada uno de los encuentros que haya tenido el docente con el evaluador.

De igual forma, es relevante mencionar que los docentes universitarios deben conocer realmente que es una evaluación de desempeño y el propósito involucrado en ella, para que puedan valorar la relevancia de este proceso. De allí que, deben estar equilibrado de que deben tener conocimientos y responsabilidad para realizar sus actividades, las cuales obedecen a una planificación previa y requiere de una inspección y control constante de los objetivos propuestos.

Por otro lado, los evaluadores universitarios deben considerar que la virtud y la validez de las funciones realizadas pueden quebrantar en la evaluación de desempeño, por ende, se debe buscar las estrategias adecuadas que permita que el proceso de evaluación deje de ser un evento difícil y tedioso, esto es posible con la formación y la información adecuada a todos los involucrado para la evaluación de desempeño.

Para resumir, la evaluación de desempeño debe apoderarse de un ambiente de trabajo acorde a las necesidades del evaluador y evaluado, para mejorar su desempeño al ejecutar el proceso y obtener mejores resultados en benefició de los involucrado desde un contexto del rendimiento académico.

\section{Referencias}

Carpio, C. (2014). Desarrollo en las Organizaciones. Bogotá, Colombia: Ediciones Costa Azul.

Chiavenato, I. (2014). Administración de Recursos Humanos. 3ra. Edición. 
México: Editorial McGraw-Hill Interamericana, S.A.

David, F (2014). La Gerencia Estratégica. Santafé de Bogotá, Colombia: Fondo Editorial Legis.

Milkovich, A., \& Boudreaw, F. (2016). Administración de Recursos Humanos. México: Editorial McGraw-Hill.

Peña, J. (2017). Formación Permanente de los Docentes como Referente de la Calidad Educativa. Revista Scientific, 2(5), 125-139, e-ISSN: 2542-2987. Recuperado de:

https://doi.org/10.29394/scientific.issn.2542-2987.2017.2.5.7.125-139

Serna, H. (2008). Gerencia estratégica. Volumen 1, 10ma Edición, Colección General, ISBN: 0000005746. Bogotá, Colombia: Editorial 3R editores. 


\section{Luís Alberto Ortiz López}

e-mail: luiyialberto64@hotmail.com

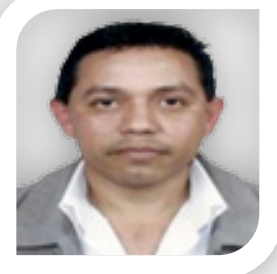

Nacido en Taborda estado Carabobo, Venezuela, el 8 de noviembre del año 1964. Postdoctorado en Filosofía y Paradigma de la Investigación en la Universidad Latinoamericana y del Caribe (ULAC); Doctor en ciencia de la educación; Magíster en gerencia y liderazgo en la educación; Licenciado en Administración de Empresas con estudios en la Universidad del Nacional Abierta (UNA), núcleo Yaracuy y la Universidad Fermín Toro (UFT); Tengo Veintiocho años de experiencia en la administración pública, en planificación y control de presupuestos; profesor de pregrado y postgrado desde hace quince años en diferentes universidades de la Ciudad de san Felipe, estado Yaracuy; En mi trayectoria profesional he sido tutor académico de trabajos de grado a nivel de pregrado y postgrado (maestría y doctorado); He participado en charlas, conversatorios y ponencias tales como la Gerencia y liderazgo participativo en la Universidad Fermín Toro (UFT), extensión San Felipe, Yaracuy, en el año 2015; Foro sobre el nuevo líder educativo del siglo XXI en la Universidad Pedagógica Experimental Libertador (UPEL), extensión San Felipe; Ponencia sobre Dinámicas Grupal como Motivación Laboral del Instituto de Previsión y Asistencia Social del Ministerio de Educación (IPASME), San Felipe; Coordinador de Danza Folklórica del IPASME, San Felipe estado Yaracuy.

El contenido de este manuscrito se difunde bajo una Licencia de Creative Commons ReconocimientoNoComercial-Compartirlgual 4.0 Internacional 